\title{
Two-Dimensional Steady-State Boundary Shape Inversion of CGM-SPSO Algorithm on Temperature Information
}

\author{
Shoubin Wang, ${ }^{1}$ Huangchao Jia, ${ }^{1}$ Xiaogang Sun, ${ }^{2}$ and Li Zhang ${ }^{1}$ \\ ${ }^{1}$ School of Control and Mechanical Engineering, Tianjin Chengjian University, Tianjin 300384, China \\ ${ }^{2}$ School of Electrical Engineering and Automation, Harbin Institute of Technology, Harbin 150001, China \\ Correspondence should be addressed to Shoubin Wang; wsbin800@126.com
}

Received 20 April 2017; Revised 4 September 2017; Accepted 19 September 2017; Published 24 October 2017

Academic Editor: Andrey E. Miroshnichenko

Copyright (c) 2017 Shoubin Wang et al. This is an open access article distributed under the Creative Commons Attribution License, which permits unrestricted use, distribution, and reproduction in any medium, provided the original work is properly cited.

\begin{abstract}
Addressing the problem of two-dimensional steady-state thermal boundary recognition, a hybrid algorithm of conjugate gradient method and social particle swarm optimization (CGM-SPSO) algorithm is proposed. The global search ability of particle swarm optimization algorithm and local search ability of gradient algorithm are effectively combined, which overcomes the shortcoming that the conjugate gradient method tends to converge to the local solution and relies heavily on the initial approximation of the iterative process. The hybrid algorithm also avoids the problem that the particle swarm optimization algorithm requires a large number of iterative steps and a lot of time. The experimental results show that the proposed algorithm is feasible and effective in solving the problem of two-dimensional steady-state thermal boundary shape.
\end{abstract}

\section{Introduction}

As a branch of the inverse problem of heat transfer, inverse geometrical problem [1] of heat conduction has a broad application prospect in industrial equipment testing, nondestructive testing [2], geometry optimization [3], biological lesions [4], and other fields. Two-dimensional steady-state space is first discretized in inverse geometrical boundary of the heat transfer system, and discretization methods mainly include finite difference method [5] (FDM) and finite element method [6] (FEM) and boundary element method [7] (BEM), which can be used for solving heat transfer problems, inverting the geometric boundary through various optimization techniques on the basis of solving the forward problem. Inversion methods based on optimization techniques can be divided into gradient-based optimization algorithms and nongradient optimization algorithms. Gradient optimization algorithm mainly includes the conjugate gradient method [8] (CGM), Levenberg-Marquardt method [9] (L-MM) and the steepest descent method [9] (SDM), and nongradient optimization algorithm mainly includes genetic algorithm [10] (GA), neural network algorithm [11] (NNM), particle swarm optimization (PSO) [12], and so forth.
CGM, L-MM, SDM, and other gradient-based optimization algorithms are the main algorithms to study the inverse problem of heat transfer and are applied to the study of geometric inverse problems of heat transfer. Carey et al. [13] studied the parallel conjugate gradient solution of the sparse system produced by the least squares combined finite element method, which showed that the algorithm was suitable for convection diffusion equation and fixed Navier-Stokes equations. Tarzanagh et al. [14] proposed a new derivative-free preconditioned conjugate gradient method to solve largescale square and undetermined nonlinear equations. The numerical results of this method for some square and undervalued test systems showed the efficiency and effectiveness, and new application was also provided for solving nonlinear differential equations.

Sellami and Chaib [15] proposed a new conjugate gradient method for unconstrained optimization, which included two existing nonlinear conjugate gradient methods that have a decrease in each iteration search direction and global convergence. Huang and Chao [16] used BEM and CGM to study the geometrical problems of heat transfer. The problem of dynamic identification of irregular boundary shape in twodimensional multiconnected region and the identification of 
TABLE 1: The number of iterations to convergence using SPSO.

\begin{tabular}{lccccccc}
\hline Iteration & 1 & 2 & 3 & 4 & 5 & 6 & 7 \\
\hline Convergence & 0.0325 & 0.0160 & 0.0140 & 0.0140 & 0.0120 & 0.0120 & 0.0120 \\
\hline
\end{tabular}

TABLE 2: The number of iterations to convergence using CGM.

\begin{tabular}{lcccccccccccc}
\hline Iteration & 1 & 2 & 3 & 4 & 5 & 6 & 7 & 8 & 9 & 10 & 11 & 12 \\
\hline Convergence & 0.1 & 0.08 & 0.068 & 0.04 & 0.025 & 0.015 & 0.008 & 0.006 & 0.005 & 0.003 & 0.003 & 0.003 \\
\hline
\end{tabular}

irregular geometric boundary with time in two-dimensional unsteady heat conduction problem were studied. BEM and CGM were used to optimize the shape of linear and nonlinear fins. The fin shape was designed according to the specific rib efficiency under the condition of certain volume of fins. The results confirmed efficiency of CGM in the equipment shape optimization.

Bin and LinHua $[17,18]$ used BEM and CGM to study the geometrical shape of the inner wall of the two-dimensional cylinder and discussed the influence of the initial stochastic value, the measurement information error, and the number of measuring points on the inversion results of the inner wall shape. Fan et al. [19] used FEM and CGM to invert the internal defects of the two-dimensional pipeline and discussed the effects of initial values and measurement errors on the inversion results. Tanweer et al. [20] proposed the problem of self-regulating particle swarm optimization (SRPSO), which achieves faster convergence and provides better solutions. Hong et al. [21-24] optimized the PSO algorithm, which can search for more simultaneous solutions and make it possible to give an unbiased estimate that provides a better way to find the global optimal solution in search space. Yuan et al. [25] introduced the improved algorithm of genetic algorithm, particle swarm optimization in two-dimensional steady-state thermal boundary problem.

CGM [26] is currently used in a more extensive inversion algorithm, which has been used in the inverse problem of heat transfer. However, gradient-based optimization algorithm belongs to the local search algorithm, which is easy to fall into the local extreme value. The inversion result has serious dependencies on the initial guess of the geometric boundary. More importantly, since the inverse problem of heat transfer, including the geometric inverse problem of heat transfer, is a typical ill-posed problem, when the temperature measurement information is not complete or there is a large measurement error, the inversion results obtained based on the gradient optimization method may deteriorate. In addition, when the geometric shapes to be inverted are more complex or the number of inversion points is larger, the calculation of the gradient matrix is time-consuming and difficult, which directly affects the engineering application of the gradient optimization method.

PSO [27] is an intelligent nongradient optimization algorithm, which is a global search algorithm with good adaptability. However this method is a global search, so the computation is relatively large, and search process is time-consuming, which result in very large restrictions in practical application of geometric inverse problem of heat transfer, especially when the inversion parameters are increased, and the shortcomings of the performance are more serious. In particular, when the measurement information is incomplete or there is a large measurement error, the inversion results obtained according to such intelligent optimization methods will also have some gaps, and the inversion method lacks the necessary antimisalignment.

In this paper, a hybrid algorithm combining conjugate gradient method $[28,29]$ and social particle swarm optimization (CGM-SPSO) algorithm is proposed to solve the problem of boundary shape recognition. In the CGM-SPSO algorithm, particle swarm optimization algorithm is used to obtain the smooth estimation of the shape by using fewer boundary elements. As the initial guess value of the conjugate gradient method, the boundary element is applied in the conjugate gradient method to ensure the accuracy of the solution. In this paper, the influence of the number of inversion parameters on the inversion solution is discussed. The convergence of CGM and SPSO algorithm in CGM-SPSO algorithm is analyzed (see Tables 1 and 2).

\section{Two-Dimensional Steady-State Thermal Conductivity Model and Its Forward Problem}

2.1. Two-Dimensional Steady-State Thermal Conductivity Model. A two-dimensional nonthermal source-dependent steady-state thermal conductivity model [25] for the detection of inner wall defects in industrial equipment is shown in Figure 1, where region is a two-dimensional space set of $x=0.0, x=10.0, y=0$, and $y=f(x)$. Here, both sides of $x=0.0$ and $x=10.0$ are adiabatic, and a known constant heat flow $q 0$ is passed out by cooling at $y=0$ so that a constant known temperature $T 0$ is maintained at $y=f(x)$.

The temperature distribution $Y_{i}$ at $y=0$ can be measured by infrared scanning thermometer. The purpose is to realize the fault detection of the inner wall of the industrial thermal equipment by reverse deduction of shape $f(x)$ of the inner wall defect. The mathematical model is shown in Figure 1.

Thermal equation in $\Omega$ region is

$$
\frac{\partial^{2} T}{\partial x^{2}}+\frac{\partial^{2} T}{\partial y^{2}}=0
$$

and when $x=0$ and $x=10$, thermal equation is

$$
\frac{\partial T}{\partial x}=0
$$




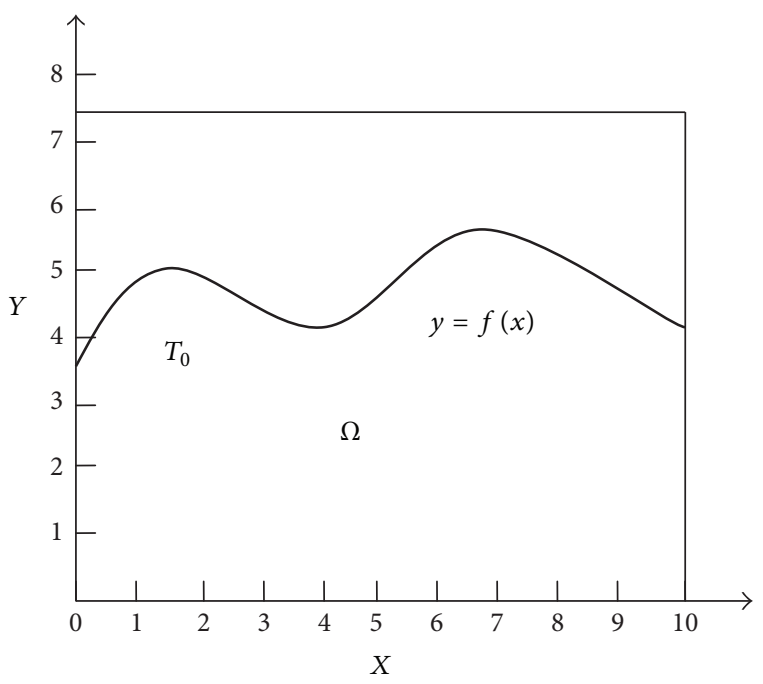

FIGURE 1: Physical model of inner wall defect detection of thermal equipment.

and when $y=0$, thermal equation is

$$
-\frac{\partial T}{\partial y}=q_{0}
$$

and when $y=f(x)$, thermal equation is

$$
\begin{aligned}
T & =T_{0}, \\
T\left(x_{i}, 0\right) & =Y_{i} \quad(i=1,2, \ldots, m) .
\end{aligned}
$$

This is a two-dimensional steady-state boundary-shaped thermal inversion problem for a known boundary temperature measurement of several boundary points and to solve the unknown boundary shape $f(x)$ of the other boundary. The solution of the inverse problem can be mathematically transformed into the optimal control problem of the following functional variants:

$$
J(f(x))=\left\|T_{i}-Y_{i}\right\|^{2}=\sum_{i=1}^{m}\left[T_{i}(x, 0)-Y_{i}(x, 0)\right]^{2},
$$

where $Y_{i}(x, 0)$ is the temperature value actually measured at the measurement point of the model surface and $T_{i}(x, 0)$ is the temperature value at the measurement point calculated from the above equations according to the guessed boundary shape.

The iterative method is used to search for the boundary problem of $f(x)$ involving the standard problem of iterative stoppage and temperature measurement error. If no measurement error exists, the stop criterion is

$$
J\left[f^{k+1}(x)\right]<\varepsilon,
$$

where $\varepsilon$ is a lesser number, such as 0.01 , which is determined by the specific convergence. If there is a measurement error and the standard deviation of the temperature measurement is $\sigma$, then the minimum criterion is determined according to the error principle.

$$
\varepsilon=3 \sigma \text {. }
$$

2.2. Boundary Element Analysis of Two-Dimensional Planar Steady Heat Conduction Problems. Boundary element method (BEM) is a computational method based on the classical integral equation, which absorbs the discrete technique of finite element method. The basic idea of the boundary element method is to use the integral equation method to solve the differential equation. The boundary element method is based on the establishment of the boundary integral equation. The solution process can be divided into two steps: the first step is the boundary of the problem, the Green formula is applied, and the differential equation in the solution domain is transformed into the integral equation on the boundary by the basic solution. The second step is the discretization of the boundary.

2.2.1. Boundary Integral Equations for Mixed Boundary Conditions. Considering the difference of boundary conditions in practical problems, two-dimensional steady-state thermal boundary conditions are taken as examples. As shown in Figure 2, boundary element analysis is carried out. Without considering the heat source, the governing equation is the Laplace equation.

In the $\Omega$ area,

$$
\nabla^{2} T=0
$$

On the boundary $\Gamma_{1}$,

$$
T=\bar{T}
$$

On the boundary $\Gamma_{2}$,

$$
q=\frac{\partial T}{\partial n}=\overline{q^{\prime}}
$$

On the boundary $\Gamma_{3}$,

$$
\frac{\partial T}{\partial n}+\frac{h}{\lambda} T=\overline{q^{\prime \prime}}
$$

In the above formula: $\Gamma=\Gamma_{1}+\Gamma_{2}+\Gamma_{3}, \overline{q^{\prime \prime}}=(h / \lambda) T_{f}$, and $T_{f}$ is the ambient temperature.

The weighting function is introduced for Laplace's equation of the above control equation $T^{*}$, applying the weighted margin method available:

$$
\begin{aligned}
\int_{\Omega}\left(\nabla^{2} T\right) T^{*} d \Omega= & \int_{\Gamma_{2}}\left(\frac{\partial T}{\partial n}-\overline{q^{\prime}}\right) T^{*} d \Gamma \\
& +\int_{\Gamma_{3}}\left(\frac{\partial T}{\partial n}+\frac{h}{\lambda} T-\overline{q^{\prime \prime}}\right) T^{*} d \Gamma \\
& -\int_{\Gamma_{1}}(T-\bar{T}) \frac{\partial T^{*}}{\partial n} d \Gamma .
\end{aligned}
$$




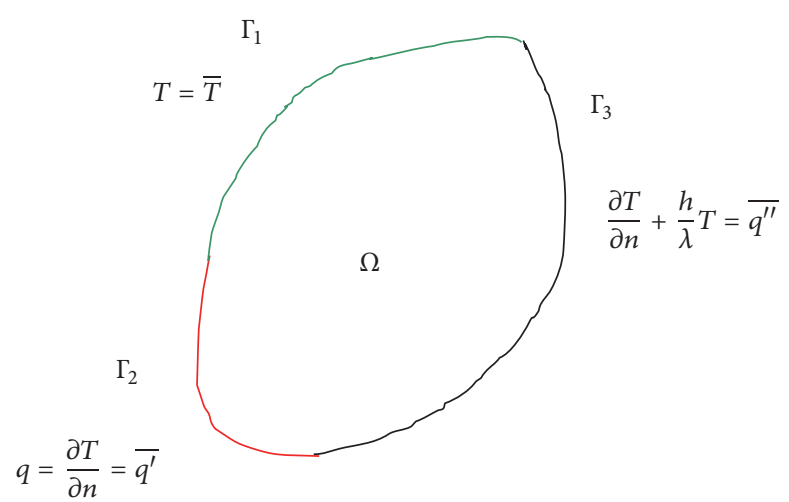

FIGURE 2: Mixed boundary conditions.

Conducting the second division points and applying Green's function for (9) available,

$$
\begin{aligned}
\int_{\Omega} T^{*}\left(\nabla^{2} T\right) d \Omega= & \int_{\Omega} \nabla\left(T^{*} \nabla T\right) d \Omega \\
& -\int_{\Omega} \nabla T \cdot \nabla T^{*} d \Omega \\
= & \int_{\Gamma} \nabla T \cdot T^{*} d \Gamma-\int_{\Omega} \nabla T \cdot \nabla T^{*} d \Omega \\
= & \int_{\Gamma} \frac{\partial T}{\partial n} \cdot T^{*} d \Gamma-\int_{\Omega} \nabla T \cdot \nabla T^{*} d \Omega \\
\int_{\Omega} \nabla T \cdot \nabla T^{*} d \Omega= & \int_{\Omega} \nabla\left(T \cdot \nabla T^{*}\right) d \Omega \\
& -\int_{\Omega} T \cdot \nabla^{2} T^{*} d \Omega \\
= & \int_{\Gamma} T \cdot \frac{\partial T^{*}}{\partial n} d \Gamma-\int_{\Omega} T \cdot \nabla^{2} T^{*} d \Omega .
\end{aligned}
$$

Substituting equations (10) into equation (9),

$$
\begin{aligned}
\int_{\Omega} T & \cdot \nabla^{2} T^{*} d \Omega+\int_{\Gamma_{1}} \frac{\partial T}{\partial n} T^{*} d \Gamma+\int_{\Gamma_{2}} \overline{q^{\prime}} T^{*} d \Gamma \\
& +\int_{\Gamma_{3}} \overline{q^{\prime \prime}} T^{*} d \Gamma \\
= & \int_{\Gamma_{1}} \bar{T} \frac{\partial T^{*}}{\partial n} d \Gamma+\int_{\Gamma_{2}} T \frac{\partial T^{*}}{\partial n} d \Gamma+\int_{\Gamma_{3}} T \frac{\partial T^{*}}{\partial n} d \Gamma \\
& +\int_{\Gamma_{3}} \frac{h}{\lambda} T T^{*} d \Gamma .
\end{aligned}
$$

Make $q=\partial T / \partial n$, and then $q^{*}=\partial T^{*} / \partial n$, On type (11) consolidation can be obtained:

$$
\begin{gathered}
\int_{\Omega} T \cdot \nabla^{2} T^{*} d \Omega+\int_{\Gamma} q T^{*} d \Gamma \\
=\int_{\Gamma} T q^{*} d \Gamma+\int_{\Gamma_{3}} \frac{h}{\lambda} T T^{*} d \Gamma .
\end{gathered}
$$

The weight function in (12) is the basic solution of Laplace's equation, which is

$$
\nabla^{2} T^{*}+\delta\left(r-r_{i}\right)=0
$$

where the point " $i$ " represents the point source point, we get formula (13) into formula (12) and use the nature of the function $\delta$, and we can get

$$
T_{i}+\int_{\Gamma} T q^{*} d \Gamma+\int_{\Gamma_{3}} \frac{h}{\lambda} T T^{*} d \Gamma=\int_{\Gamma} q T d \Gamma .
$$

This is the boundary integral equation for the inner nodes of any point in the $\Omega$ domain.

Moving the point " $i$ " to the boundary and getting the boundary integral equation at any point on the boundary,

$$
\frac{1}{2} T_{i}+\int_{\Gamma} T q^{*} d \Gamma+\int_{\Gamma_{3}} \frac{h}{\lambda} T T^{*} d \Gamma=\int_{\Gamma} q T^{*} d \Gamma .
$$

Formula (14) and formula (15) can be combined to obtain the boundary integral equation of any point in the domain $\Omega$ and, at any point on the boundary,

$$
C_{i} T_{i}+\int_{\Gamma} T \cdot q^{*} d \Gamma+\int_{\Gamma_{3}} \frac{h}{\lambda} \cdot T^{*} \cdot T d \Gamma=\int_{\Gamma} q \cdot T^{*} d \Gamma,
$$

where, in area domain $\Omega, C_{i}=1$ and, on the border $\Gamma, C_{i}=$ $1 / 2$.

The weight function $T^{*}$ is the fundamental solution of the two-dimensional Laplace equation:

$$
T^{*}=\frac{1}{2 \pi} \ln \frac{1}{r} .
$$

2.2.2. Boundary Discrete Equations for Mixed Boundary Conditions. Formula (16) is discretized, and the boundary is divided into $N$ units. On each boundary element, $T$ and $q$ can be uniformly interpolated by ordinary unit, linear unit, or quadratic unit according to different interpolation to form matrix:

$$
\sum_{j=1}^{N} H_{i j} T_{j}=\sum_{j=1}^{N} G_{i j} q_{j}
$$


where

$$
H T=G q
$$

When $i=j$, then $H_{i i}=C_{i}+\widehat{H}_{i i}$; when $i \neq j$, then $H_{i j}=\widehat{H}_{i j}$; and when " $j$ " falls $\Gamma_{3}$, then $H_{i j}=H_{i j}+(h / \lambda) \cdot G_{i j}$; moving the unknown amount to the right and moving the known amount to the left,

$$
A X=F .
$$

Solution for the above equations can be obtained in $T$ and $q$ in the unknown amount.

\section{Inverse Problem}

3.1. Improved Particle Swarm Optimization Algorithm. All the individual optimal solutions pbest (best particle) in particle swarm optimization algorithm are connected with the population optimal solution gbest (best group), which is a typical star structure with the center node gbest. In this structure, each individual unconditionally follows the population optimal, so that the individual optimal can easily converge to the population optimal. When the gbest is the local optimal, the particle swarm algorithm will fall into the local optimal. Thus, an improved strategy is proposed named social particle swarm optimization (SPSO). By setting a different harsh threshold for each individual, one determines whether the individual is following other individuals, or is maintaining the current state, or is free to move, in order to maintain the diversity of individuals in the population, to avoid the algorithm precocious and into the local optimum.

In the social particle swarm algorithm, the characteristics of social atoms are introduced into the particle swarm algorithm, and each individual is given a skeleton threshold, where the individual with a threshold of 0 means that the movement is not affected by any other individual information, and the threshold with a threshold of nonzero may have different attraction points in the process of population updating to maintain the current state, so the social particle swarm algorithm may have different attraction points in the process of updating the population according to the new attracting point.

There are two types of particles in the social particle swarm algorithm: free particles and followers. The free particle is a particle with a threshold of zero, which is not affected by the behavior of other particles and randomly determines the next generation particle position. The follower particle with a nonzero value is affected by the attraction point during the search process. Whether or not to follow the attraction point depends on how many other particles follow. The updating formula of follower particle in SPSO algorithm is

$$
\begin{aligned}
v_{i j}(t+1)= & w v_{i j}(t)+c_{1} r_{1}\left(\text { pbest }_{i j}(t)-x_{i j}(t)\right) \\
& +c_{2} r_{2}\left(\operatorname{attract}_{i j}(t)-x_{i j}(t)\right), \\
x_{i j}(t+1)= & x_{i j}+v_{i j}(t+1),
\end{aligned}
$$

where the third term in (21) is changed from the standard gbest $_{i j}$ in PSO to the attraction point attract ${ }_{i j}$, and it may be different for different particles. When the algorithm is initialized, the individual with the best fitness is selected as the initial attraction point in the particle selection population. As the search progresses, each free particle is likely to become a new attraction point, and if there is a fitness for a free particle that is better than the fitness of all other particles, it becomes a new attraction. Individuals with a threshold of 1 in the particle are first attracted, and then the individual with higher thresholds will also move toward the attraction point, and the individuals whose attraction population does not reach the threshold will remain unchanged.

The implementation steps of social particles swarm algorithm are shown as follows.

Step 1 (algorithm initialization). The population size $N$, the largest evolution algebra Gen, acceleration factors $c 1, c 2$, and inertia weight $w$ are given in advance. Give each particle a follow threshold, which is taken as an integer in $[0, N]$. The optimal individual in initial population is set to attraction point.

Step 2. Update the speed and position of the following particles according to (21) and (22), and free particles randomly generate the next generation position.

Step 3. Calculate the fitness $f\left(x_{i+1}\right)$ of all individuals.

Step 4. Update optimal positions pbest of all individuals.

Step 5. If the optimal fitness value of free particle is better than the population optimal fitness value, then update attracting point attraction equal to pbest, which indicates the free individual is more attractive and will attract the individual of getting the threshold. These attractive points that follow the individual will be updated to the optimal position of the free particle individual, and the attraction point of the other unattended individuals will remain unchanged.

Step 6. Repeat Steps 2 to 5 until the algorithm termination condition is met.

The flow chart of SPSO algorithm is shown in Figure 3.

3.2. Conjugate Gradient Method. The conjugate gradient [25] transforms the inverse problem into three problems: direct question, sensitive problem, and concomitant problem. The problem is based on the assumed boundary shape (1d) and other boundary conditions, using the boundary element method to solve (1a)-(1e), then calculating the minimum value of the objective function (2). The circulation mode of conjugate gradient method is

$$
f^{n+1}(x)=f^{n}(x)-\beta^{n} p^{n}(x), \quad n=0,1,2, \ldots,
$$

where $\beta^{n}$ is the search step of $n$ to $n+1$ and $p^{n}(x)$ is the search direction:

$$
p^{n}(x)=J^{\prime n}(x)+\gamma^{n} p^{n-1}(x),
$$

where $J^{\prime n}(x)$ is gradient direction, so the $n$th search direction $p^{n}(x)$ is the conjugate of gradient direction $J^{\prime n}(x)$ and search 


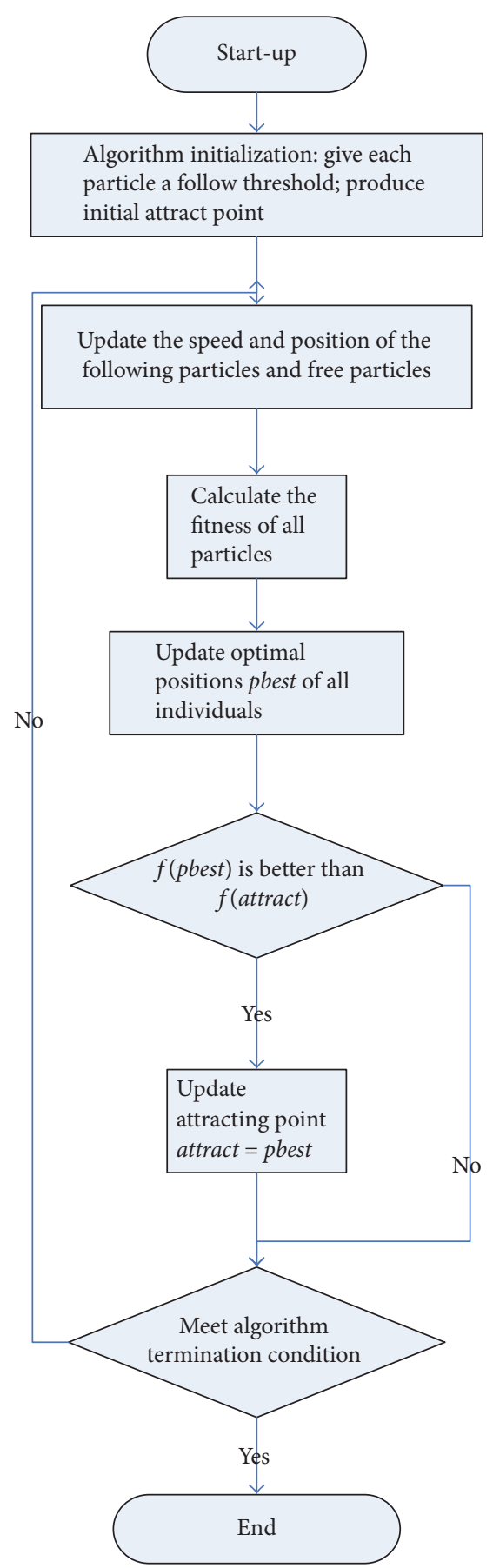

FIGURE 3: SPSO algorithm flow chart.

direction $p^{n-1}(x)$ of $n-1 . \gamma^{n}$ is conjugate coefficient, which can be calculated by the equation

$$
\gamma^{n}=\frac{\int_{x=0}^{L}\left(J^{\prime n}\right)^{2} d x}{\int_{x=0}^{L}\left(J^{\prime n}-1\right)^{2} d x},
$$

where $\gamma^{0}=0$.
3.2.1. Sensitivity Problem. For the inverse problem of heat transfer, the existence of solutions can be determined from the physical problem; for example, for a transient problem, the temperature distribution has changed, which must be caused by a change in the parameters, such as boundary conditions and geometric conditions. For some individual antirecognition problems, we can also prove the uniqueness of the strict solution. Although the validity of the solution 
must be judged from the physical problem for most problems, the biggest problem of the inverse problem is that when the temperature distributed measurement results with some error may cause some parameters of the anticalculation results with unpredictable errors. Therefore, in the process of calculation, we must use some technical means to ensure the stability of the results, so as to detect whether an inverse problem calculation method is effective but also to discuss the calculation results on the temperature measurement error sensitivity. So the conjugate gradient needs to solve the problem of sensitivity when solving the inverse problem.

The determination of the search step in (23) needs to find out a sensitivity problem called the increment problem. The sensitivity problem refers to the variation of the change amount $\Delta T(x)$ of surface temperature $T$ when the boundary shape $f(x)$ has an increment $\Delta f(x)$, and the formula is

$$
\left(\frac{\partial J}{\partial f}\right)^{T}=\left(\frac{\partial J}{\partial f_{1}}, \frac{\partial J}{\partial f_{2}}, \ldots, \frac{\partial J}{\partial f_{n}}\right) .
$$

The specific approach is as follows: in the formula (1a)-(1e), $T+\Delta T$ replaces the original $T$, and $f(x)+\Delta f(x)$ replaces the original $f(x)$. And then subtract the original equations to get the sensitivity problem, so, in the region $\Omega$, the thermal equation is

$$
\frac{\partial^{2} \Delta T}{\partial x^{2}}+\frac{\partial^{2} \Delta T}{\partial y^{2}}=0
$$

When $x=0$, the thermal equation is

$$
\frac{\partial \Delta T}{\partial x}=0
$$

When $x=10$, the thermal equation is

$$
\frac{\partial \Delta T}{\partial x}=0
$$

When $y=0$, the thermal equation is

$$
\frac{\partial \Delta T}{\partial y}=0
$$

When $y=f(x)$, the thermal equation is

$$
\Delta T=\Delta f \frac{\partial T}{\partial y}
$$

Similarly, the boundary element method is used to discretize the above equations and obtain the temperature increment $\Delta T$. According to formula (2), iteration $J\left(f^{n+1}\right)$ of $n+1$ can be written:

$$
J\left(f^{n+1}\right)=\sum_{i=1}^{m}\left[T_{i}\left(f^{n}-\beta^{n} p^{n}\right)-Y_{i}\right]^{2} .
$$

Take Taylor expansion $T_{i}\left(f^{n}-\beta^{n} p^{n}\right)$ and take out two linear terms, and the following equation can be drawn:

$$
J\left(f^{n+1}\right)=\sum_{i=1}^{m}\left[T_{i}\left(f^{n}\right)-\beta^{n} \Delta T_{i}\left(p^{n}\right)-Y_{i}\right]^{2} .
$$

Seeking derivative for $\beta^{n}$ and making it zero, the search step is available:

$$
\beta^{n}=\frac{\sum_{i=1}^{m}\left(T_{i}-Y_{i}\right) \Delta T_{i}}{\sum_{i=1}^{m}\left(\Delta T_{i}\right)^{2}}
$$

3.2.2. Adjoint Problems. In formula (23), the new boundary shape function $f^{n+1}(x)$ is calculated according to the boundary function $f^{n}(x)$ of the last iteration, and the search direction $p^{n}(x)$ is also needed to be found out. Therefore, the gradient direction $J^{\prime n}(x)$ is needed to be calculated according to (24), which is the so-called functional derivative problem, known as the adjoint problem. In order to derive the equation of the adjoint direction, we need to multiply the basic control formula (1a) by a Lagrangian operator $\lambda(x, y)$ (also called adjoint function) and integrate the spatial domain and then add the result to the right of (2). The expression of the function $J[f(x)]$ is as follows:

$$
\begin{aligned}
J[f(x)]= & \int_{x=0}^{L}[T-Y]^{2} \delta\left(x-x_{i}\right) d x \\
& +\int_{x=0}^{L} \int_{y=0}^{f(x)} \lambda\left[\frac{\partial^{2} T}{\partial x^{2}}+\frac{\partial^{2} T}{\partial y^{2}}\right] d y d x .
\end{aligned}
$$

With $T+\Delta T$ instead of $T$ and $f+\Delta f$ instead of $f$, the following are obtained after a series of arrangement:

$$
\int_{a}^{b}[T-Y]^{2} d x=\int_{a}^{b} \int_{0}^{L}[T-Y]^{2} \delta\left(x-x_{i}\right) d x d y .
$$

Then

$$
\begin{aligned}
\Delta J & =J(q+\Delta q)-J(q) \\
& =\int_{x=0}^{L} 2(T-Y) \Delta T \delta\left(x-x_{i}\right) d x \\
& +\int_{x=0}^{L} \int_{y=0}^{f(x)} \lambda\left[\frac{\partial^{2} \Delta T}{\partial x^{2}}+\frac{\partial^{2} \Delta T}{\partial y^{2}}\right] d y d x .
\end{aligned}
$$

Make the second distribution equal to integral twice in the right side of (33), and use the boundary conditions of sensitivity problem, then make $\Delta J$ closer to 0 , and finally adjoint problems can be as follows.

In the region $\Omega$,

$$
\frac{\partial^{2} \lambda}{\partial x^{2}}+\frac{\partial^{2} \lambda}{\partial y^{2}}=0
$$

When $x=0$,

$$
\frac{\partial \lambda}{\partial x}=0
$$

When $x=10$,

$$
\frac{\partial \lambda}{\partial x}=0
$$


When $y=0$,

$$
\frac{\partial \lambda}{\partial y}=-2(T-Y) \delta\left(x-x_{i}\right) .
$$

When $y=f(x)$,

$$
\lambda=0 \text {. }
$$

The boundary element method is used to solve the corresponding value of the adjoint function $\lambda(x, y)$, so that the functional increment is

$$
\Delta J=\int_{0}^{L}-\left[\frac{\partial \lambda}{\partial y} \cdot \frac{\partial T}{\partial y}\right]_{y=f(x)} \Delta f(x) d x
$$

So according to Alifanov's definition,

$$
\Delta J=\int_{0}^{L} J^{\prime}(x) \Delta f(x) d x .
$$

The derivative of the functional $J$ is

$$
J^{\prime}(x)=-\left.\frac{\partial \lambda}{\partial y} \cdot \frac{\partial T}{\partial y}\right|_{y=f(x)} .
$$

The above three problems including the forward problem, the sensitivity problem, and the adjoint problem can be solved, and the following are iterative calculation step of conjugate gradient method:

(1) Select a stochastic initial value of inner wall shape in $f(x)$ to solve the forward problem (1a)-(1e) and calculate the temperature distribution $T(x, t)$ in the domain.

(2) According to $T_{i}$ and $Y_{i}$ determine whether to meet the convergence stop standard (3) $J\left[f^{k+1}(x)\right]<\varepsilon$; if satisfied, then stop the iteration; otherwise, calculate the next step.

(3) Solve the adjoint problem (34a)-(34e), and get the adjoint function $\lambda(x)$.

(4) Calculate the gradient $J^{\prime}(x)$.

(5) Calculate the conjugate gradient coefficient $\gamma^{k}$ and the descent direction $p^{k}$.

(6) Make $\Delta f(x)=-p^{k}(x)$ to solve the sensitivity problem (27a)-(27e) to get the temperature increment $\Delta T(x)$.

(7) Calculate the search step $\beta^{k}$ by formula (30).

(8) Make $k=k+1$ to calculate the new boundary shape $f^{k}(x)$ and new $T_{i}$ and go back to step (2).

3.3. CGM-SPSO Hybrid Algorithm. CGM is computationally fast, but it usually converges to local optimal solutions and relies heavily on the initial approximation of the iterative process. The SPSO algorithm requires tens of thousands of iterations and a lot of computation time. The fitness function evaluation of a complex problem is very time-consuming, so we propose a hybrid algorithm of CGM and SPSO, whose

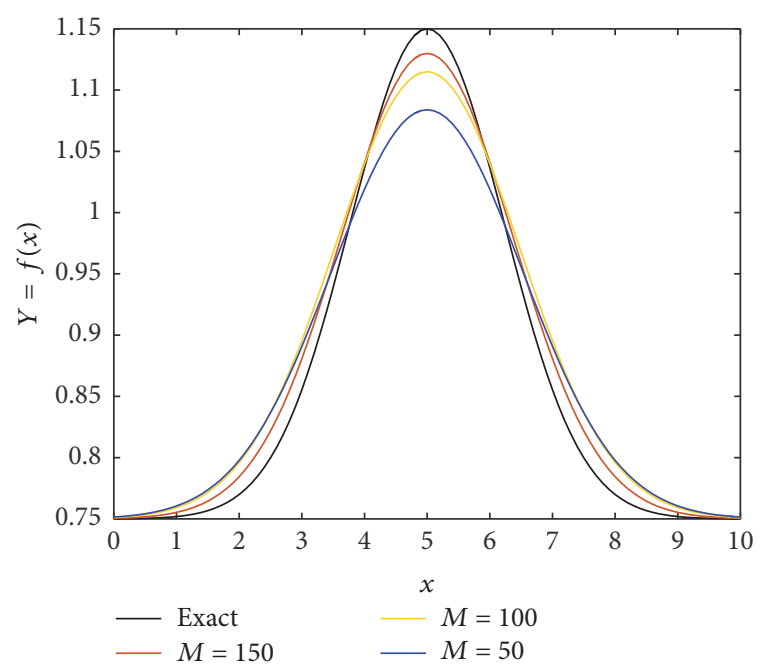

FIGURE 4: Inverse solution of different geometric inversion parameters number.

purpose is to combine global search ability of SPSO and fast convergence ability of CGM. Since CGM needs a smooth and appropriate initial value, it can converge to a very precise solution. Therefore, this paper gets the temperature of the measured point $T\left(T_{1}, T_{2}, \ldots, T_{i}\right)$ by the SPSO method when the boundary shape is unknown, and then the value obtained by SPSO is processed by the interpolation calculation to obtain a smooth function as the initial value of CGM.

\section{Exponential Analysis}

As shown in Figure 1, the physical model of the inner wall defect detection of industrial thermal equipment is a twodimensional thermostatic heat conduction problem. The domain $\Omega$ is a two-dimensional space composed of $x=0.0$, $x=10.0, y=0$, and $y=f(x)$. Here, both sides of $x=0.0$ and $x=10.0$ are adiabatic, and a known constant heat flow $q 0$ is passed out by cooling at $y=0$ so that a constant known temperature $T 0$ is maintained at $y=f(x)$.

4.1. Influence of the Number of Geometric Inversion Parameters. Assume the function of the thermal device boundary is expressed as

$$
f(x)=0.75+0.4 \exp \left(-\frac{(x-5)^{2}}{3}\right) .
$$

Considering the uniform distribution of the temperature measurement points in the outer boundary of the thermal equipment, there is no measurement error. Three geometric inversion parameters $M=50, M=100$, and $M=150$ are geometrically inversed. The inversion results are shown in Figure 4.

The experimental data for each group are as follows. 
When $M=50$,

1 to 13 columns

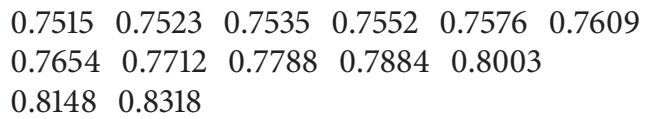

14 to 26 columns

$\begin{array}{llllll}0.8515 & 0.8738 & 0.8982 & 0.9242 & 0.9512 & 0.9782\end{array}$ $\begin{array}{llllll}1.0042 & 1.0282 & 1.0490 & 1.0656 & 1.0772 & 1.0832\end{array}$ 1.0833

27 to 39 columns

$\begin{array}{llllll}1.0774 & 1.0659 & 1.0494 & 1.0286 & 1.0047 & 0.9787\end{array}$ $\begin{array}{lllllll}0.9517 & 0.9247 & 0.8987 & 0.8742 & 0.8519 & 0.8322\end{array}$ 0.8151

40 to 50 columns

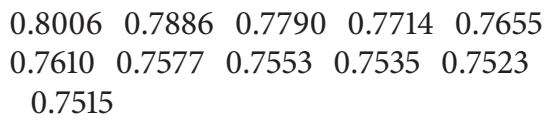

When $M=100$,

1 to 13 columns

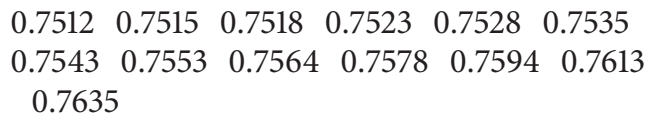

14 to 26 columns

$\begin{array}{llllll}0.7660 & 0.7690 & 0.7724 & 0.7762 & 0.7806\end{array}$

$\begin{array}{lllllll}0.7856 & 0.7912 & 0.7974 & 0.8043 & 0.8119 & 0.8203\end{array}$ 0.82940 .8393

27 to 39 columns

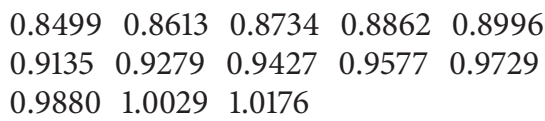

40 to 52 columns

$\begin{array}{llllll}1.0318 & 1.0453 & 1.0581 & 1.0698 & 1.0805 & 1.0900\end{array}$ $\begin{array}{llllll}1.0980 & 1.1046 & 1.1097 & 1.1131 & 1.1148 & 1.1148\end{array}$ 1.1131

53 to 65 columns

$\begin{array}{llllll}1.1097 & 1.1047 & 1.0981 & 1.0900 & 1.0806 & 1.0699\end{array}$ $\begin{array}{lllllll}1.0582 & 1.0454 & 1.0319 & 1.0177 & 1.0031 & 0.9881\end{array}$ 0.9730

66 to 78 columns

$\begin{array}{llllll}0.9579 & 0.9428 & 0.9281 & 0.9137 & 0.8997 & 0.8863\end{array}$ $\begin{array}{llll}0.8735 & 0.8614 & 0.8500 & 0.8394\end{array}$

$\begin{array}{llll}0.8295 & 0.8204 & 0.8120\end{array}$
79 to 91 columns

$$
\begin{array}{llllll}
0.8044 & 0.7975 & 0.7912 & 0.7856 & 0.7807 & \\
0.7763 & 0.7724 & 0.7690 & 0.7661 & 0.7635 & 0.7613 \\
0.7594 & 0.7578 & & & &
\end{array}
$$

92 to 100 columns

$$
\begin{array}{llllll}
0.7564 & 0.7553 & 0.7543 & 0.7535 & 0.7528 & 0.7523 \\
0.7518 & 0.7515 & 0.7512 & & &
\end{array}
$$

When $M=150$,

1 to 13 columns

$$
\begin{array}{llllll}
0.7505 & 0.7506 & 0.7507 & 0.7508 & 0.7510 & 0.7511 \\
0.7514 & 0.7516 & 0.7519 & 0.7522 & 0.7526 & 0.7530 \\
0.7535 & & & & &
\end{array}
$$

14 to 26 columns

$$
\begin{array}{lllll}
0.7540 & 0.7547 & 0.7554 & 0.7562 & 0.7571 \\
0.7582 & 0.7594 & 0.7607 & 0.7622 & 0.7638 \\
0.7656 & 0.7677 & 0.7699 & &
\end{array}
$$

27 to 39 columns

$$
\begin{array}{llllll}
0.7724 & 0.7751 & 0.7782 & 0.7814 & 0.7850 & 0.7889 \\
0.7932 & 0.7977 & 0.8027 & 0.8080 & & \\
0.8137 & 0.8198 & 0.8263 & & &
\end{array}
$$

40 to 52 columns

$$
\begin{array}{lllll}
0.8332 & 0.8405 & 0.8482 & 0.8563 & 0.8648 \\
0.8736 & 0.8829 & 0.8925 & 0.9024 & 0.9126 \\
0.9231 & 0.9339 & 0.9448 & &
\end{array}
$$

53 to 65 columns

$\begin{array}{llllll}0.9559 & 0.9671 & 0.9783 & 0.9896 & 1.0008 & 1.0120\end{array}$ $\begin{array}{llllll}1.0229 & 1.0337 & 1.0441 & 1.0543 & 1.0640 & 1.0732\end{array}$ 1.0820

66 to 78 columns

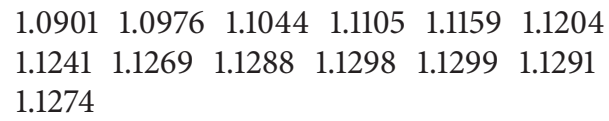

79 to 91 columns

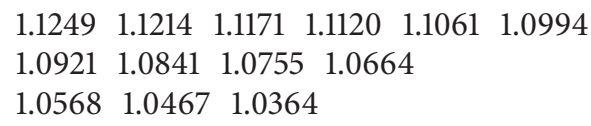

92 to 104 columns

$$
\begin{array}{llllll}
1.0257 & 1.0148 & 1.0037 & 0.9925 & 0.9812 & 0.9699 \\
0.9587 & 0.9476 & 0.9366 & 0.9258 & 0.9153 \\
0.9050 & 0.8950 & & & &
\end{array}
$$


105 to 117 columns

$$
\begin{array}{lllll}
0.8853 & 0.8759 & 0.8670 & 0.8584 & 0.8502 \\
0.8424 & 0.8350 & 0.8280 & 0.8214 & 0.8152 \\
0.8094 & 0.8040 & 0.7990 & &
\end{array}
$$

118 to 130 columns

$$
\begin{array}{lllll}
0.7943 & 0.7900 & 0.7860 & 0.7823 & 0.7790 \\
0.7759 & 0.7731 & 0.7705 & 0.7682 & 0.7661 \\
0.7642 & 0.7626 & 0.7610 & &
\end{array}
$$

131 to 143 columns

$$
\begin{array}{lllll}
0.7597 & 0.7585 & 0.7574 & 0.7564 & 0.7556 \\
0.7548 & 0.7542 & 0.7536 & 0.7531 & 0.7527 \\
0.7523 & 0.7519 & 0.7517 & &
\end{array}
$$

144 to 150 columns

$$
\begin{array}{lllll}
0.7514 & 0.7512 & 0.7510 & 0.7509 & 0.7507 \\
0.7506 & 0.7505 & & &
\end{array}
$$

As can be seen from Figure 4, when using the CGMSPSO hybrid algorithm for geometric inversion, regardless of whether the number of inversion parameters is $M=50, M=$ 100 , or $M=150$, the estimated boundary shape and the exact boundary shape are very close, and this happens equally in the edge and peak. The average relative errors of the inversion are $1.12 \%, 0.73 \%$, and $0.52 \%$, respectively, which shows CGM-SPSO algorithm is good to overcome the influence of the number of inversion parameters on the exact value of the inversion. It can be seen from the above experiments that when the number of geometric inversion parameters increases, the inversion solution accuracy is improved, but it can be seen from the figure that increasing the number does not have much effect on the accuracy of the inversion solution. Therefore, the conclusion is that the proposed method is not affected by the influence of the number of inversion parameters on the inversion solution. The following discusses the convergence of SPSO and CGM when the number of inversion parameters is $M=100$, as shown in Figures 5 and 6.

As shown in Figures 5 and 6, the convergence curve of the SPSO algorithm shows that the objective function converges rapidly after the fifth iteration. Then, CGM obtains initial value from SPSO. After 10 iterations, a convergent optimal solution is produced. It shows that CGM-SPSO algorithm spends the less computation time and has not only high accuracy of the solution but also the higher efficiency of the algorithm.

\section{Conclusion}

In this paper, a hybrid algorithm of conjugate gradient method and particle swarm optimization algorithm is used to study the inversion problem of the boundary shape. The social particle swarm optimization algorithm and the conjugate gradient method are introduced, and the twodimensional steady-state model is used to verify and discuss

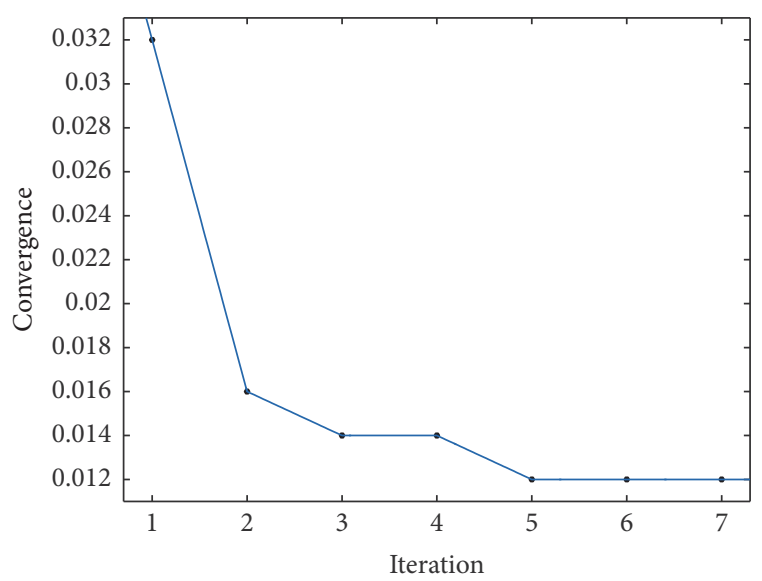

FIGURE 5: Convergence curve of SPSO algorithm.

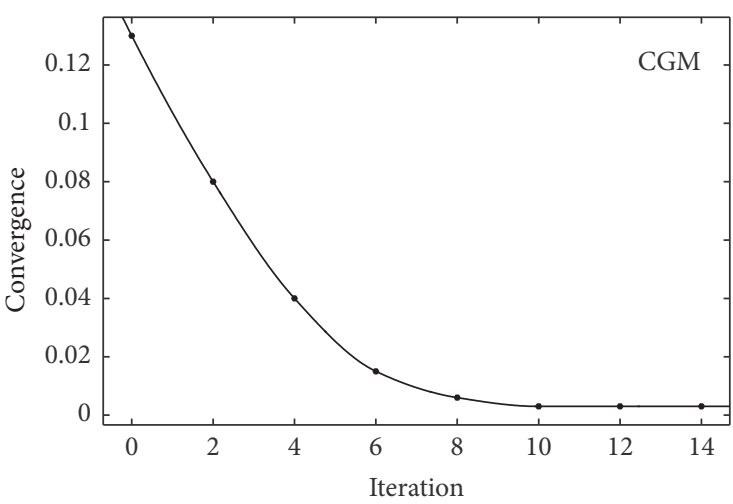

FIGURE 6: Convergence curve of CGM algorithm.

the algorithm. The influence of the number of inversion parameters on the inversion results is discussed, and the convergence of the SPSO algorithm and the CGM algorithm is obtained. The main conclusions are as follows.

(1) The CGM-SPSO algorithm is used to reconstruct the geometric shape of the two-dimensional steady-state thermal conduction system. When the number of geometric inversion parameters increases, the inversion solution is improved, but when the number of geometric inversion parameters is appropriate, increasing the number does not have much effect on the inversion solution. It is proved that CGM-SPSO is effective for two-dimensional steady-state thermal boundary identification.

(2) CGM-SPSO algorithm has high accuracy to the peak and edge value of geometric shape.

(3) Less times of iterations can meet the convergence conditions; thus calculation time is greatly reduced.

\section{Conflicts of Interest}

The authors declare no conflicts of interest. 


\section{Authors' Contributions}

Shoubin Wang and Huangchao Jia contributed to developing the ideas of this research. Shoubin Wang and Xiaogang Sun performed this research. All of the authors were involved in preparing this manuscript. Shoubin Wang and Huangchao Jia wrote the paper.

\section{Acknowledgments}

This work was financially supported by the National Key Foundation for Exploring Scientific Instrument of China (2013YQ470767), Tianjin Science and Technology Committee for Science and Technology Development Strategy Research Project (15ZLZLZF00350), Tianjin Science and Technology Commissioner Project (16JCTPJC53000), and the 13th Five-Year Plan (2016-2020) of Science Education Project in Tianjin city (HE1017).

\section{References}

[1] S. Wang, L. Zhang, X. Sun, and H. Jia, "Solution to two-dimensional steady inverse heat transfer problems with interior heat source based on the conjugate gradient method," Mathematical Problems in Engineering, Art. ID 2861342, 9 pages, 2017.

[2] C.-R. Su and C.-K. Chen, "Geometry estimation of the furnace inner wall by an inverse approach," International Journal of Heat and Mass Transfer, vol. 50, no. 19-20, pp. 3767-3773, 2007.

[3] C. Huang, C. C. Chiang, S. K. Chou et al., "Inverse geometry designproblem in optimizing hull surfaces," Journal of ship research, vol. 42, no. 2, pp. 79-85, 1998.

[4] P. W. Partridge and L. C. Wrobel, "An inverse geometry problem for the localisation of skin tumours by thermal analysis," Engineering Analysis with Boundary Elements, vol. 31, no. 10, pp. 803-811, 2007.

[5] H. Fu, M. K. Ng, and H. Wang, "A divide-and-conquer fast finite difference method for space-time fractional partial differential equation," Computers and Mathematics with Applications, 2016.

[6] A. Løkke and A. K. Chopra, "Direct finite element method for nonlinear analysis of semi-unbounded dam-water-foundation rock systems," Earthquake Engineering \& Structural Dynamics, vol. 46, no. 8, pp. 1267-1285, 2017.

[7] M. Feischl, "Adaptive boundary element methods for optimal convergence of point errors," Numerische Mathematik, vol. 132, no. 3, pp. 541-567, 2016.

[8] Z. Zhao, X.-Q. Jin, and Z.-J. Bai, "A geometric nonlinear conjugate gradient method for stochastic inverse eigenvalue problems," SIAM Journal on Numerical Analysis, vol. 54, no. 4, pp. 2015-2035, 2016.

[9] K. Zhou, J. Hou, H. Fu, B. Wei, and Y. Liu, "Estimation of relative permeability curves using an improved LevenbergMarquardt method with simultaneous perturbation Jacobian approximation," Journal of Hydrology, vol. 544, pp. 604-612, 2017.

[10] B. A. Norman and J. C. Bean, "A genetic algorithm methodology for complex scheduling problems," Naval Research Logistics (NRL), vol. 46, no. 2, pp. 199-211, 1999.

[11] M. Ghaedi, "Artificial neural network and bees algorithm for removal of eosin B using cobalt oxide nanoparticle-activated carbon: isotherm and kinetics study," Environmental Progress \& Sustainable Energy, vol. 34, no. 1, pp. 155-168, 2015.

[12] S. Zhang, "Optimal computing budget allocation for particle swarm optimization in stochastic optimization," IEEE Transactions on Evolutionary Computation, pp. 1-1, 2016.

[13] G. F. Carey, Y. Shen, and R. T. Mclay, "Parallel conjugate gradient performance for least-squares finite elements and transport problems," International Journal for Numerical Methods in Fluids, vol. 28, no. 10, pp. 1421-1440, 1998.

[14] D. A. Tarzanagh, P. Nazari, and M. R. Peyghami, "A nonmonotone PRP conjugate gradient method for solving square and under-determined systems of equations," Computers \& Mathematics with Applications, 2016.

[15] B. Sellami and Y. Chaib, "A new family of globally convergent conjugate gradient methods," Annals of Operations Research, vol. 241, no. 1-2, pp. 497-513, 2016.

[16] C.-H. Huang and B.-H. Chao, "An inverse geometry problem in identifying irregular boundary configurations," International Journal of Heat and Mass Transfer, vol. 40, no. 9, pp. 2045-2053, 1997.

[17] L. Bin and L. LinHua, "A Geometric boundary identification algorithm for thermal problem based on boundary element discretization," Journal of China Electromechanical Engineering, vol. 20, no. 1, pp. 38-43, 2008.

[18] L. Bin, Boundary Element Method for Geometric Inverse Problem of Thermal Conductivity, Harbin Institute of Technology, China, 2008.

[19] C. Fan, F. Sun, and L. Yang, "An algorithm study on the identification of a pipeline's irregular inner boundary based on thermographic temperature measurement," Measurement Science and Technology, vol. 18, no. 7, pp. 2170-2177, 2007.

[20] M. R. Tanweer, S. Suresh, and N. Sundararajan, "Self regulating particle swarm optimization algorithm," Information Sciences, vol. 294, pp. 182-202, 2015.

[21] X. Hong and L. Panchi, "Improved quantum behavior particle swarm optimization algorithm and its application," Information and control, vol. 02, pp. 157-164, 2016.

[22] F. Wei, S. Jun, X. Zhenping et al., "Convergence analysis and control parameters of quantum particle swarm optimization algorithm," Journal of Physics, vol. 59, no. 6, pp. 3686-3694, 2010.

[23] L. Weitian, Research on Improved Particle Swarm Optimization Algorithm and Its Application, East China University of Science and Technology, China, 2014.

[24] T. Na, Research and Application of Numerical Solution of Inverse Problem for Partial Differential Equation, Jiangnan University, China, 2012.

[25] G. Yuan, Z. Meng, and Y. Li, "A modified Hestenes and Stiefel conjugate gradient algorithm for large-scale nonsmooth minimizations and nonlinear equations," Journal of Optimization Theory and Applications, vol. 168, no. 1, pp. 129-152, 2016.

[26] Y. Zhang, H. Zheng, and C. Zhang, "Global convergence of a modified PRP conjugate gradient method," in Proceedings of the 1st International Conference on Advances in Computational Modeling and Simulation 2011, ACMS 2011, pp. 986-995, chn, December 2011.

[27] H. Zhu, Y. Wang, K. Wang, and Y. Chen, "Particle Swarm Optimization (PSO) for the constrained portfolio optimization problem," Expert Systems with Applications, vol. 38, no. 8, pp. 10161-10169, 2011. 
[28] H. Fu and H. Rui, "A priori error estimates for least-squares mixed finite element approximation of elliptic optimal control problems," Journal of Computational Mathematics, vol. 33, no. 2, pp. 113-127, 2015.

[29] Y. Zhang, "Preconditioned Bi-conjugate gradient method of large-scale sparse complex linear equation group," Chinese Journal of Electronics, vol. 20, no. 1, pp. 192-194, 2011. 

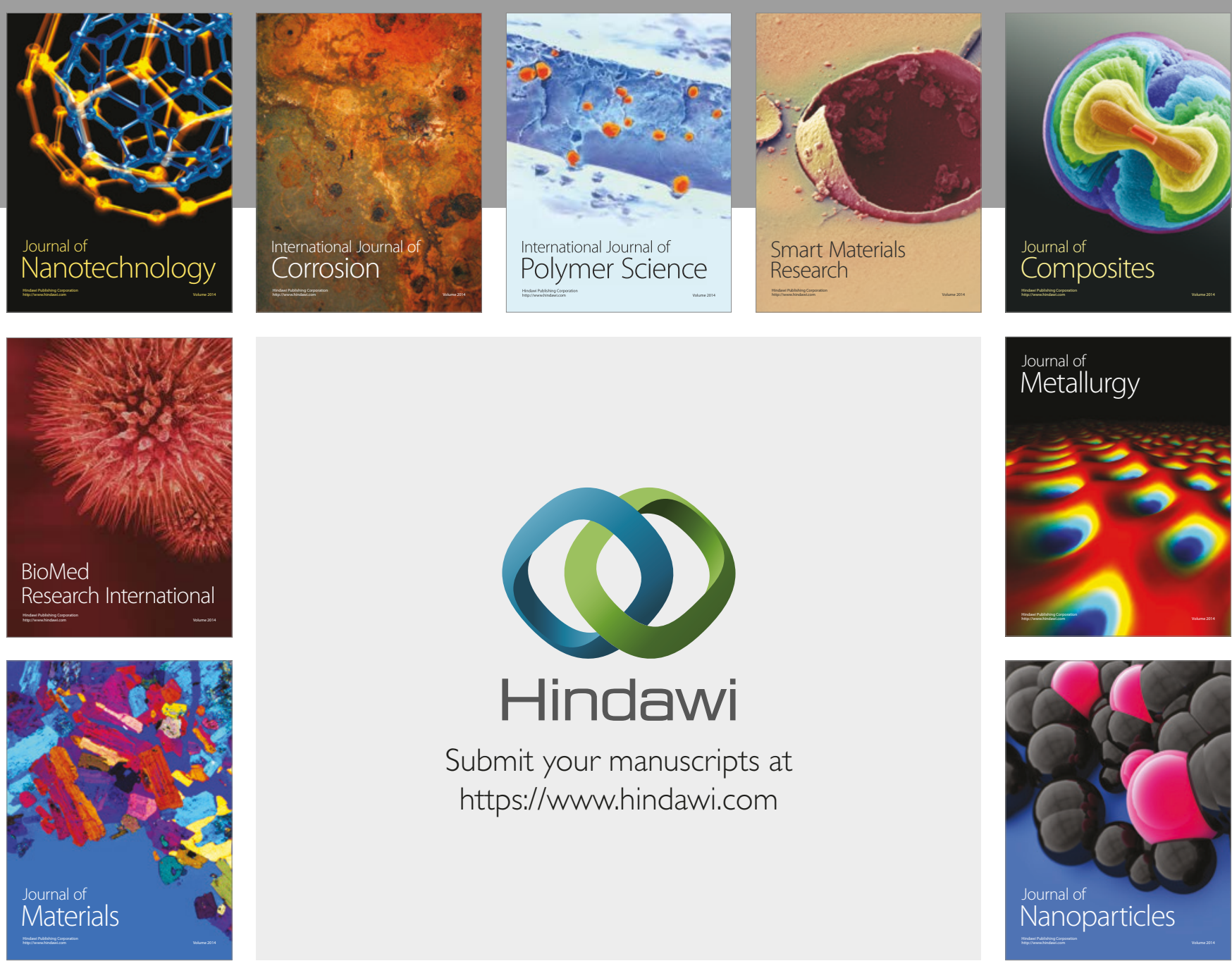

\section{Hindawi}

Submit your manuscripts at

https://www.hindawi.com
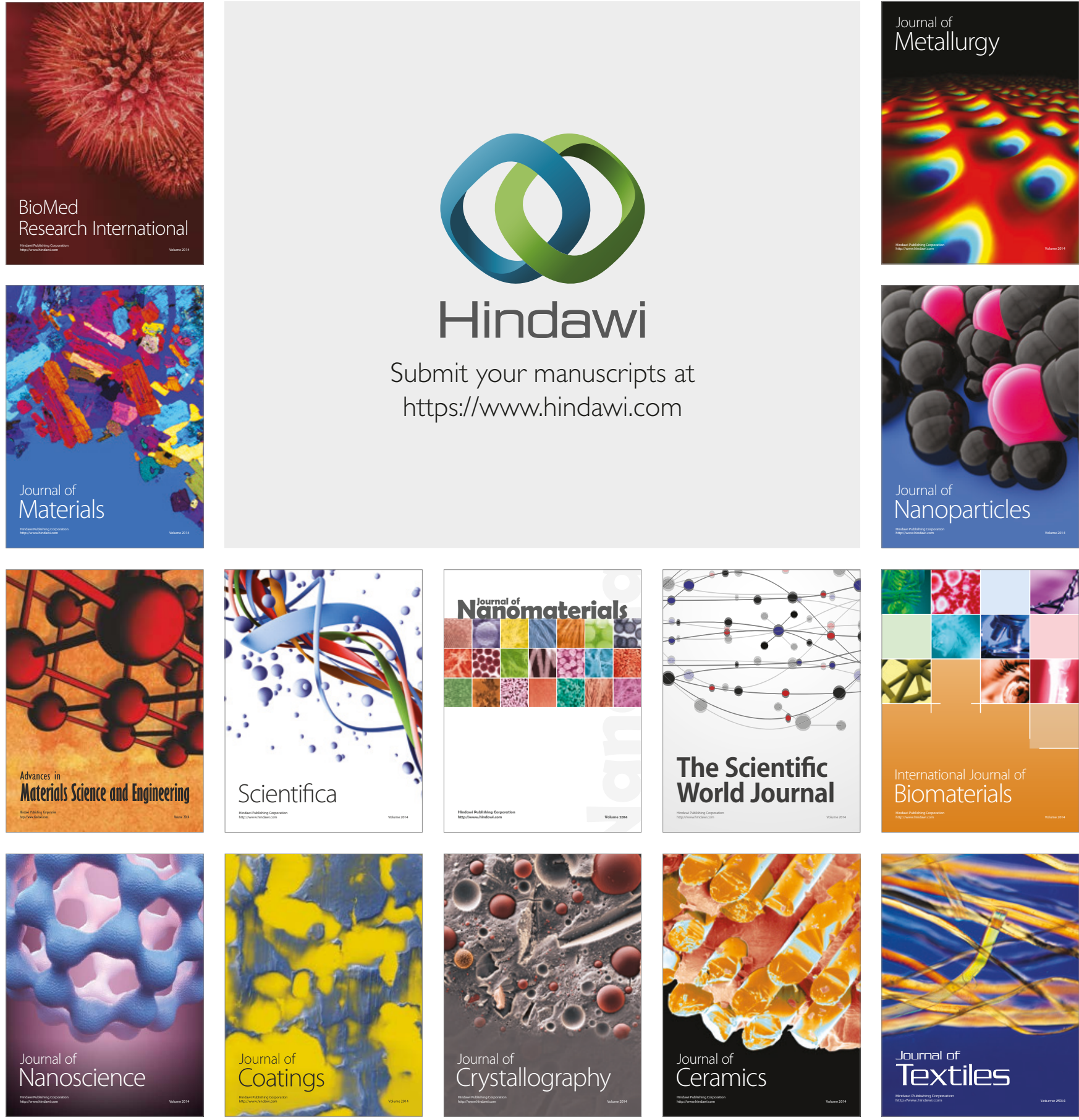

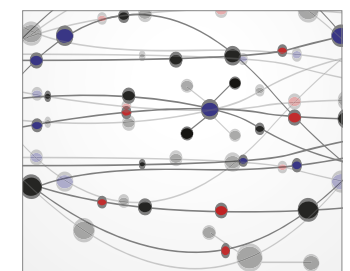

The Scientific World Journal
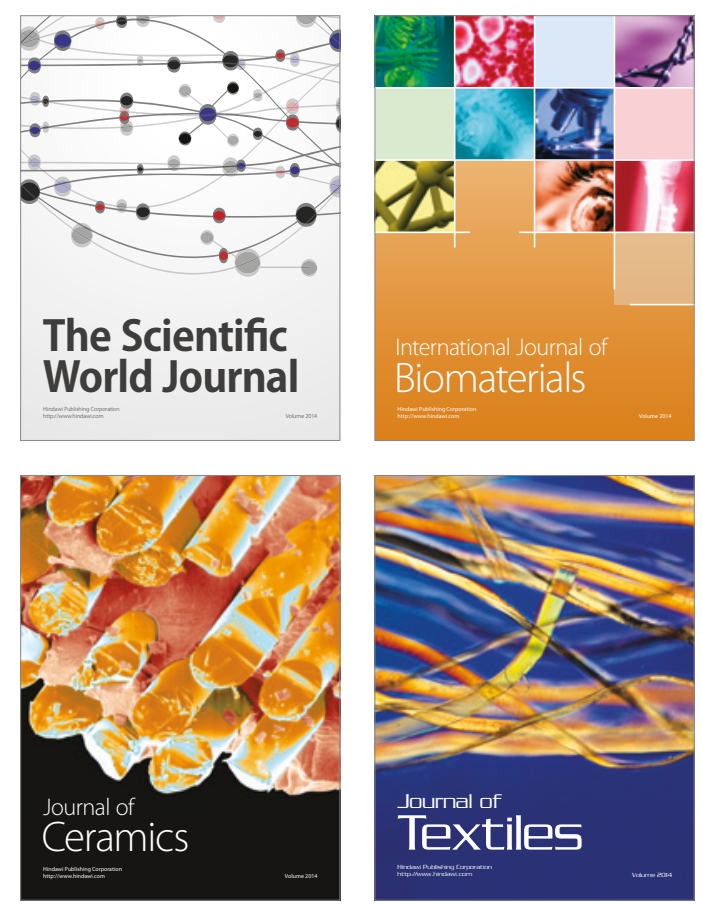